\section{Ginecologia \\ MENINGIOMA INTRACRANIANO E USO DE ESTERÓIDES SEXUAIS EM MULHERES}

Blitshteyn S et al.' em análise retrospectiva de dez anos, avaliaram 350.000 prontuários eletrônicos de mulheres entre 26 e 86 anos que procuraram atendimento médico por qualquer queixa clínica; constataram que I.390 eram de mulheres com história de meningiomas sintomáticos ou incidentalmente descobertos (meningioma silencioso) e, que II\% (156) das I.390 eram usuárias ou tinham usado previamente terapêutica hormonal $(\mathrm{TH})$.

Os resultados de regressão logística ajustados para a idade mostraram associação positiva entre o uso de TH e o diagnóstico de meningioma $[\mathrm{OR}=2.2(95 \% \mathrm{Cl}, \mathrm{I} .9$ to $2.6 ; \mathrm{P}<.000 \mathrm{I})]$ e desvelaram uma freqüência de meningioma nas usuárias de $\mathrm{TH}$ atuais ou prévias de 865/100.000, diferentemente dos 366/100.000 nas não-usuárias.

\section{Comentário}

Investigações moleculares e clínicas sugerem um importante papel dos hormônios esteróides sexuais femininos na ocorrência de meningiomas; de fato, isso se torna mais plausivel ao se constatar que os meningiomas são mais prevalentes em mulheres, exibem crescimento durante a gestação e são coincidentes com o câncer de mama, permitindo reforçar a participação do fator endócrino na eclosão desses tumores.

Contudo, os dados da literatura são limitados e controversos não só no tocante ao uso da terapia hormonal (TH), mas também dos contraceptivos orais (CO) como possíveis fatores de risco para meningiomas.

Do exposto, então se depreende a enorme dificuldade do médico em decidir sobre o início ou manutenção da TH ou do CO em mulheres com diagnóstico presumível ou conhecido de meningioma intracraniano, questão muito comum em neurocirurgia.

Preocupados com o tema, em 2006 Custer et al. ${ }^{2}$, em estudo caso-controle, avaliaram a possibilidade de associação entre CO ou TH com meningioma; nessa investigação, o diagnóstico de meningioma foi confirmado por histopatologia e também foram avaliados a presença de receptores de estrogênios (RE) e de progesterona (RP).

Os resultados evidenciaram discreto aumento no risco de meningioma em usuárias de $\mathrm{CO}$, sem relação com a duração do uso; da mesma forma, as ex-usuárias de TH exibiram também modesto incremento do risco, enquanto nas usuárias atuais nenhum risco foi observado. O estudo dos receptores mostrou que dos 142 espécimes disponíveis, somente dois casos (1\%) expressaram RE, enquanto 130 (92\%) o fizeram para RP; o uso de CO associou-se a um maior risco de meningioma em mulheres com menor expressão do $R P$, enquanto que o uso de
TH após a menopausa conferiu efeito protetor não significativo e independente da expressão de RP.

Em 2007, Claus EB et al. ${ }^{3}$, ainda inquietos com o tema, elaboraram uma revisão sistemática e identificaram sete publicações; seis foram elegíveis pelos critérios de inclusão, sendo que cinco eram estudos do tipo caso-controle, um retrospectivo e nenhum randomizado. Concluíram que não há evidência estatística de aumento do risco de meningioma em usuárias de $\mathrm{CO}$, porém sugeriram uma associação do risco naquelas em uso de $\mathrm{TH}$.

A análise atenta e crítica desses estudos permitem-nos concluir que:

1. Há evidências de associação positiva entre TH e o risco de meningioma e que seu uso pode ser um fator de risco para o tumor;

2. Os COS parecem não aumentar o risco de menigioma;

3. Outros estudos são necessários para melhor esclarecer a relação entre esteróides sexuais exógenos e meningioma e devem enfocar a idade de uso, os tipos de hormônios prescritos, a duração de uso e a avaliação do tipo de receptor esteroídico presente no meningioma.

José M. Aldrighi

Alessandro Scapinell

SONIA TAMANAHA

тsтоми Аокı

Referências:

I. Blitshteyn S, Crook JE, Jaeckle KA. Is there an association between meningioma and hormone replacement therapy? J Clin Oncol. 2008;26(2):279-82.

2. Custer B, Longstreth WT Jr, Phillips LE, Koepsell TD, Van Belle G. Hormonal exposures and the risk of intracranial meningioma in women: a populationbased case-control study. BMC Cancer. 2006;6(I): I 52.

3. Claus EB, Black PM, Bondy ML, Calvocoressi L, Schildkraut JM, Wiemels JL, Wrensch $M$. Exogenous hormone use and meningioma risk: what do we tell our patients? Cancer. 2007; I I0(3):47| -6.

\section{Clinica Ciriengica}

\section{EFEITO DA DERIVAÇÃO GÁSTRICA NO ESÔFAGO DE BARRETT E NA METAPLASIA INTESTINAL DA CÁRDIA EM PACIENTES OBESOS MÓRBIDOS}

Os autores analisaram, em estudo prospectivo, 557 pacientes obesos mórbidos submetidos à gastroplastia aberta entre agosto de 1999 e outubro de 2004. Todos os pacientes foram submetidos à endoscopia e biopsias pré-operatórias. Dos 557 pacientes, I 5 apresentavam metaplasia intestinal no esôfago distal ou na cárdia. Todos foram submetidos à endoscopia pré-operatoria e, no mínimo, a duas endoscopias pós-operatórias. Em todas as endoscopias eram realizadas quatro biópsias nos quatro quadrantes do epitélio de Barrett. Os pacientes foram divididos em dois grupos: Barrett curto (até $30 \mathrm{~mm}$ ) e Barrett longo (mais de $31 \mathrm{~mm}$ ). Todos os 
pacientes foram submetidos à cirurgia de Fobi-Capella por via laparotomica.

Resultados: $\bigcirc$ sintoma de refluxo gastroesofágico desapareceu em todos os pacientes, e 12 meses após a cirurgia, a EDA mostrou cicatrização das ulceras pépticas em esôfago e da esofagite erosiva. A análise histológica das biópsias mostrou que dos sete pacientes com Barrett curto, em quatro (57\%) houve desaparecimento da metaplasia intestinal em até 25 meses após a cirurgia. Dos cinco pacientes com Barrett longo, um (20\%) apresentou regressão da metaplasia intestinal. Em nenhum paciente houve aparecimento de displasia nos tecidos biopsiados. Dois pacientes que apresentavam displasia leve no pré-operatório tiveram desaparecimento da displasia com retorno a epitélio normal no pós-operatório e um paciente apresentou regressão da displasia permanecendo apenas com metaplasia intestinal.

Dos três pacientes com metaplasia intestinal em cárdia, dois apresentaram regressão para mucosa normal e um permaneceu com metaplasia, no entanto sem progressão para displasia.

Conclusão: A cirurgia de derivação gástrica para tratamento da obesidade mórbida é uma ótima cirurgia antirrefluxo, levando a um total desaparecimento dos sintomas de refluxo gastroesofágico, a cicatrização de úlceras de esôfago e esofagite erosiva. Além disso, a cirurgia levou a uma regressão da metaplasia intestinal para mucosa normal em grande número de pacientes, principalmente no Barrett curto. Houve desaparecimento da displasia e, no caso de persistência da metaplasia, não houve aparecimento de displasia no período de seguimento endoscópico pós-operatório de dois anos.

\section{Comentário}

A cirurgia de Fobi-Capella leva a uma diminuição da produção ácida no estômago (pequena câmara) e evita o refluxo duodenal. Esses dois fatores diminuem o risco de Barrett, e propiciam o seu desaparecimento. Devido ao pequeno número de pacientes desse trabalho (1 5 pacientes), são necessários novos estudos com maior número de casos e com mais tempo de seguimento para conclusões definitivas sobre o assunto.

ELIAS JiRJoss ILIAS Paulo Kassab

\section{Referência}

Csendes A, Burgos AM, Smok G, Burdiles P, Henríquez A. Effect of gastric bypass on Barrett's esophagus and intestional metaplasia of the cárdia in patients with morbid obesity. Gastroint Surg 2006; $10(2): 259-64$.

\section{Emergencia e Medicina Intensiva}

\section{ALTERAÇÖES DA GLICEMIA EM TERAPIA INTENSIVA PEDIÁTRICA: UMA PREOCUPAÇÃO CRESCENTE}

Dentre as alterações endocrinológicas mais importantes observadas em crianças gravemente doentes encontram-se a insuficiência adrenal e os distúrbios da glicemia'. Estes últimos são responsáveis por grande número de publicações nos últimos anos visando a correlação entre tais distúrbios e desfechos desfavoráveis ao paciente grave.

Wintergerst et al. publicaram estudo em $2006^{2}$ analisando os efeitos tanto da hiperglicemia e hipoglicemia, mas também da variação glicêmica durante a internação, parâmetro este nunca avaliado anteriormente. $\bigcirc$ estudo foi realizado em UTI Pediátrica terciária com predomínio de pacientes cirúrgicos (cerca de 70\%) e trouxe o importante dado da mortalidade mais alta ocorrer nos pacientes hipoglicêmicos (feito corte de glicemia abaixo de $65 \mathrm{mg} / \mathrm{dL}$, porém esta UTI também contava com população considerável de recém-nascidos) - dos 50 óbitos observados na pesquisa, em 30 pacientes foram observados níveis glicêmicos abaixo de $65 \mathrm{mg} / \mathrm{dL}$. Os efeitos da hiperglicemia na mortalidade dos pacientes também foi significativa, com $68 \%$ dos óbitos ocorrendo entre pacientes com níveis glicêmicos máximos superiores a 200 mg/dL, com nível de significância "p" inferior a 0,00 I . A taxa de mortalidade foi de $5,7 \%$ em níveis glicêmicos entre I I 0 mg/dL e 150 mg/dL, 7,4\% entre 150 mg/dL e 200 mg/dL e 9,9\% quando o nível glicêmico ultrapassou $200 \mathrm{mg} / \mathrm{dL}$. Em contraste, pacientes com níveis glicêmicos normais tiveram mortalidade de apenas I,5\% como citado perfazendo apenas dois dos 50 óbitos observados no estudo. Mostra-se interessante o dado citado pelo autor que o parâmetro que mais obteve significância estatística relacionada ao aumento da mortalidade foi a variabilidade glicêmica. Nas crianças com variações grandes de glicemia, principalmente naquelas que experimentaram hipo e hiperglicemia, a mortalidade foi ainda maior do que aquelas que apresentaram algum dos dois distúrbios isoladamente.

\section{Comentário}

Existem evidências, conforme demonstrado em várias publicações recentes, de que a hiperglicemia é altamente prevalente em crianças internadas em terapia intensiva e, além disso, contribui isoladamente para o aumento da morbimortalidade durante a internação e do próprio tempo de internação. Na população adulta, tal correlação foi demonstrada há alguns anos. Van den Berghe et al. publicaram estudo pioneiro em $2001^{3}$ comprovando a efetividade na redução de quase $50 \%$ na mortalidade de pacientes adultos internados em UTI cirúrgica quando submetidos a controle estrito da glicemia com insulinoterapia contínua e, ao analisar a população não-cirúrgica cinco anos depois ${ }^{4}$, não obteve impacto na mortalidade com o mesmo modelo de terapia.

A hipoglicemia, apesar de menos prevalente de maneira isolada, obteve no estudo citado ${ }^{2}$ importante correlação com tempo de internação hospitalar e mortalidade. A hiperglicemia, do mesmo modo, também esteve correlacionada a tais desfechos. Até o presente momento, a quase totalidade dos estudos pediátricos demonstra, de maneira retrospectiva e com análise de prontuários, que tal correlação da hiperglicemia com morbimortalidade em terapia intensiva é significativa; entretan- 